\title{
Mind Controlled Devices
}

\author{
Disha Moreshiya \\ PCB Designer \\ Nagpur, India
}

\author{
Nayan Dhoke \\ Programmer \\ Nagpur, India
}

\author{
Akshy Sadawarti \\ Presentator \\ Nagpur, India
}

\author{
Parthao Lad \\ Planner \\ Nagpur, India
}

\begin{abstract}
Mind Controlled devices' this project is totally based on the mind waves that is alpha waves. That mind waves has frequency in between $7.5 \mathrm{~Hz}$ to $12.5 \mathrm{~Hz}$.All biological system is operates in same frequency range. Now this thing will have many advantages to control many different devices like AC or DC devices. This will be very use full for many fields like in defense, medical uses, industry, also in daily life. This project will increase the human limits. It also make life easy. Mind Controlled devices will consist of EEG electrodes some instrumental amplifies microcontroller boards through the instrumental amplifier we will trying to amplify the alpha signals which is read able to the microcontroller board.
\end{abstract}

Keywords: - Arduino, instrumental amplifier (INA), L293D, Controlling unit, alpha waves, mind reading, TTL Logic converter.

\section{INTRODUCTION}

Alpha waves are neural oscillations in the frequency range of 7.5-12.5 Hz arising from synchronous and coherent (in phase or constructive) electrical activity of thalamic pacemaker cells in humans. They are also called Berger's wave in memory of the founder of EEG. Alpha waves are one type of brain waves detected either by electroencephalography (EEG) or magneto encephalography (MEG) and predominantly originate from the occipital lobe during wakeful relaxation with closed eyes. Alpha waves are reduced with open eyes, drowsiness and sleep. Historically, they were thought to represent the activity of the visual cortex in an idle state. More recent papers have argued that they inhibit areas of the cortex not in use, or alternatively that they play an active role in network coordination and communication. Occipital alpha waves during periods of eyes closed are the strongest EEG brain signals.

In this Project we are using Arduino, L293D, INA332, Relay, Other things. In this we are amplifying the brain waves through INA332 then that INA 332 give the signal to Arduino now this board utilizing and identifying the purpose of the signal and converting in to movement using motor relays or any other AC or DC Devices. The brain waves will read through the EEG Electrode (Electroencephalogram).

\section{History}

1) Vladimirovich (1912) He described the human alpha and beta rhythms

First animal EEG study (dog)

1929: Hans Berger developed electroencephalography, the graphic representation of the difference in voltage between two different cerebral locations plotted over time

Cybulski (1914)

Cybulski acquired camera, which he use to perform the first electroencephalogram of the brain wave monkey
First human EEG recordings

'Invented' the term electroencephalogram (EEG)

3) American EEG Society formed in 1947

American Clinical Neurophysiology Society (ACNS) was founded in 1946, originally named the American Electroencephalographic Society (AEEGS). In 1995, AEEGS changed its name to the current designation, ACNS, to more accurately encompass the scope of its mission and goals. ACNS is the major professional organization in the United States devoted to the establishment and maintenance of standards of professional excellence in clinical neurophysiology in the practice of neurology, neurosurgery and psychiatry.

4 Aserinsky \& Kleitman (1953)

First EEG recordings of REM sleep (.75"). The text should be in two $8.45 \mathrm{~cm}(3.33 ")$ columns with a $.83 \mathrm{~cm}(.33 ")$ gutter.

Quantitative EEG (QEEG) is digital recording of the EEG. For decades it was only possible to record the various brain waves on paper with the traditional polygraph. The EEG rhythms were amplified and used to drive pens, one for each recording electrode. As the pens fluctuated from the EEG rhythms, a long piece of graph paper was dragged under the pens by a motor, creating the graph of the electrical activity on the outer surface of the brain.

Historically, they were thought to represent the activity of the visual cortex in an idle state. More recent papers have argued that they inhibit areas of the cortex not in use, or alternatively that they play an active role in network coordination and communication. Occipital alpha waves during periods of eyes closed are the strongest EEG brain signals Neural oscillations in the frequency range of $7.5-12.5 \mathrm{~Hz}$ arising from synchronous and coherent (in phase or constructive) electrical activity of thalamic pacemaker cells in humans

2) Berger (1924) 


\section{Components}

\section{1) TI Parts}

\section{i) INA332}

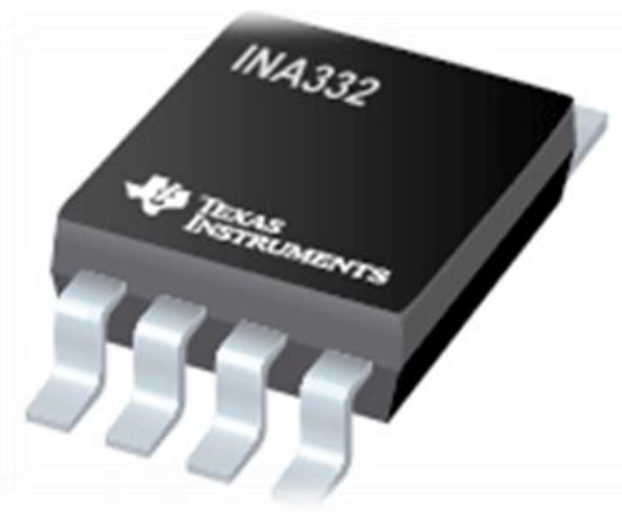

\section{Figure 1 INA332}

The INA332 and INA2332 are rail-to-rail output, low-power CMOS instrumentation amplifiers that offer wide range, single supply, and bipolar-supply operation. Using a special manufacturing flow, the INA332 family provides the lowest cost available, while still achieving low-noise amplification of differential signals with low quiescent current of $415 \mu \mathrm{A}$ (dropping to $0.01 \mu \mathrm{A}$ when shut down). Returning to normal operation within microseconds, this INA can be used for battery or multichannel applications.

\section{ii) MSP430F5529 USB Experimenter's Board}

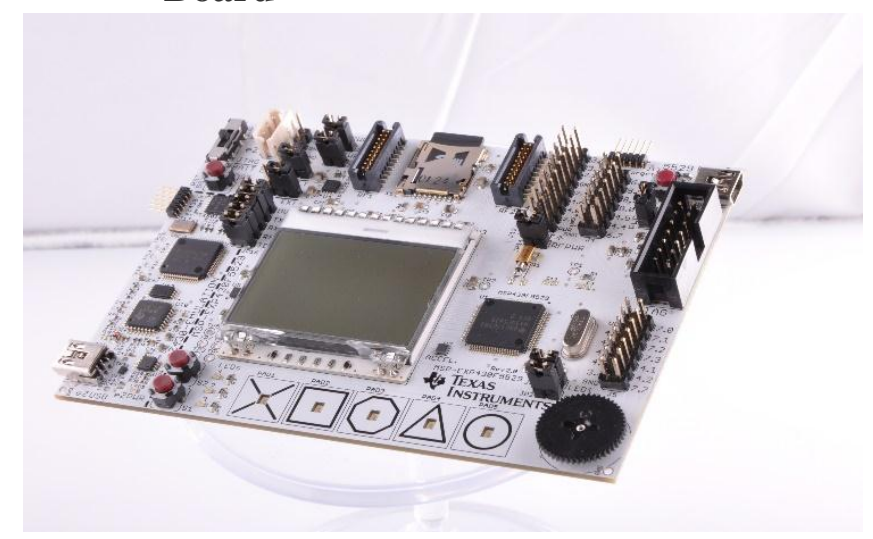

\section{$\begin{array}{llll}\text { Figure } & 2 & \text { MSP430F5529 } & \text { USB }\end{array}$ \\ Experimenter's Board}

The MSP-EXP430F5529 Experimenter Board is a development platform based on the MSP430F5529 with integrated USB. The Experimenter Board showcases the abilities of the latest family of MSP430s and is perfect for learning and developing USB-based applications using the MSP430. The features include a 102x64 dot-matrix LCD, micro SD memory card interface, 3-axis accelerometer, five capacitive-touch pads, RF EVM expansion headers, nine LEDs, an analog thumb-wheel, easy access to spare F5529 pins, integrated Spy-Bi-Wire flash emulation module, and standard full JTAG pin access. The kit is pre-programmed with an out-of-box demo to immediately demonstrate the capabilities of the MSP430 and Experimenter Board. This document details the hardware, its use, and the example software.

This equipment is intended for use in a laboratory test environment only environments may cause interference with radio communications, in which case the user, at his own expense, will be required to take whatever measures may be required to correct this interference.

\section{iii) L293D}

\section{Figure 3 L293D Motor driver IC}

1.1 The L293 and L293D are quadruple high-current half-H drivers. These devices are designed to drive a wide array of inductive loads such as relays, solenoids, DC and bipolar stepping motors, Title and Authors

\section{Block Diagram}

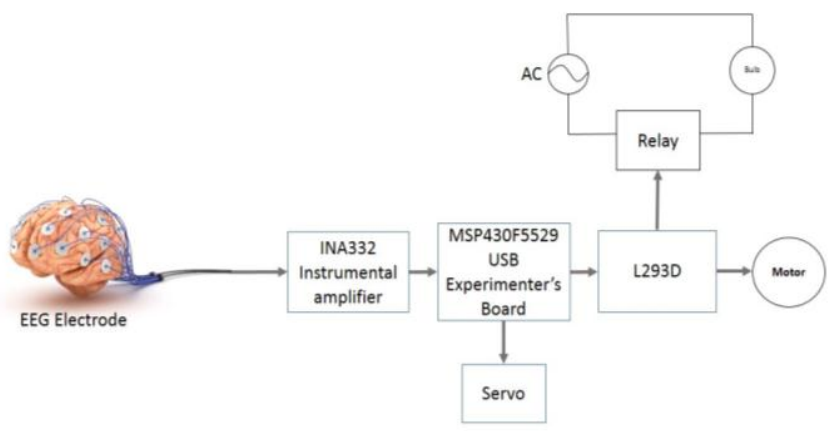

the above block diagram we are using EEG Electrode. EEG electrode receives the signals from the brain, the received signal is very low so it will be amplified by INA332. It is an instrumental amplifier which amplifies the signal and send that 
signal to MSP Board which will verify that signal and decide the purpose of the signal and give to the L293D for driving of motor or any other devices.

\section{1) INA332}

It is an instrumental amplifier which amplifies the signal coming from the electrode to readable signal for the MSP Board (Microcontroller) as shown in the above diagram.

2) MSP430F5529 USB Experimenter's Board

It consist of microcontroller, display, micro SD Card Slot, USB Development Platform, etc. MSP Board will receive that signals from INA332 then it will utilize this signal to decide the purpose of signal.

\section{3) L293D}

These devices are designed to drive a wide array of inductive loads such as relays, solenoids, DC and bipolar stepping motors, as well as other high-current and high-voltage loads.

\section{4) SERVO}

Servo will directly operate through MSP Board

\section{Innovativeness of the Proposed Solution:-}

This will be very use full for many fields like in defense, medical uses, industry, also in daily life. This project will increase the human limits. It also make life easy.

Using this concept in defense to control guns, missiles, tanks in critical condition such as war, terrorist attacks etc. We can use this product as gun controller from long range for the protection of soldier in the battle field \& we can use as targeting system.

Now a day we know about the battlefield situation \& the terrorist attack it is become very horrible \& dangerous because of the latest weapons \& advance technology.

But our product will change the scenario of the battle field.

\section{Need of this Project}

\section{1) Used for Defense:}

Using this concept in defense to control guns, missiles, tanks in critical condition such as war, terrorist attacks etc. We can use this product as gun controller from long range for the protection of soldier in the battle field \& we can use as targeting system.

Now a day we know about the battlefield situation \& the terrorist attack it is become very horrible $\&$ dangerous because of the latest weapons \& advance technology.

But our product will change the scenario of the battle field.
Patients like: - Paralyzed, Coma patients, Handicaps, Prosthetic limbs, etc.

This product can use for the all type of patients those who cannot talk, hear, and walk, blind.

If the patients is in coma the brain of that person is always working. So through this product will help to us to understand what the patient want.

For the paralyzed patient those who can't walk this product can help to drive the wheel chair for those patients using their mind.

This product can use normal person also in their daily life. For make their life easier.

We will make this product low power consumption as well as low cost. Which make this product purchasable for middle class as well as upper class customers.

\section{ACKNOWLEDGMENTS}

Our thanks to the experts who have contributed towards development of the template.

\section{REFERENCES}

1. Taken From the history that is given in the page no. $1 \& 2$ as well as from IEEE papers the relationship of alpha waves and theta waves in EEG during relaxation and IQ test http://ieeexplore.ieee.org/document/5940766/

2. History:-www.wikipidia.com

3. Figure1:http://www.braincoreofthelowcountry.com/wpcontent/uploads/2014/07/brain-4.jpg

4. Figure2:- www.neuro24.de/eeg.htm

5. Figure3:- www.neuro24.de/eeg.htm

6. Figure 4, 5, and 6:- http://www.ti.com

7. Page 2:- www.wikipidia.com

8. Page 3:- http://www.ti.com

\section{1) Use for Patients:}

